Publicacions Matemàtiques, Vol 36 (1992), 217-228.

\title{
ON HARMONIC VECTOR FIELDS
}

\author{
JERZY J. KONDERAK
}

\begin{abstract}
A tangent bundle to a Riemannian manifold carries various metrics induced by a Riemannian tensor. We consider harmonic vector fields with respect to some of these metrics. We give a simple proof that a vector field on a compact manifold is harmonic with respect to the Sasaki metric on TM if and only if it is parallel. We also consider the metrics $I I$ and $I+I I$ on a tangent bundle (cf. [YI]) and harmonic vector fields getierated by them.
\end{abstract}

\section{Preliminaries}

1.1. Let $(M, g)$ be a smooth manifold. We denote by $N:=T M$ the tangent bundle. Then there is given the canonical projection $\pi: N \rightarrow M$. By $\Gamma\left(T^{*} M\right)$ we shall denote the set of 1 -forms on $M$. Then there exists a natural map

$$
\text { i : } \Gamma\left(T^{*} M\right) \longrightarrow C^{\infty}(N)
$$

such that $(\mathrm{i} \theta)(v):=\theta(v)$ for each $\theta \in \Gamma\left(T^{*} M\right)$ and $v \in N$.

Suppose that $X \in \chi(M)$ is a vector field on $M$. Then there is defined the vertical lift $X^{V}$ of $X$ to $N$. The vector field $X^{V}$ has the property that

$$
X^{V}(\mathrm{i} \theta)=\theta(X) \circ \pi
$$

for all 1 -forms $\theta$ on $M$. Moreover it is well-known that the above equality determines uniquely the vertical lift of $X$.

Observation 1.1. The vertical lift of a vector field depends pointwisely on vectors.

Observation 1.2. Suppose that for a given $x \in M$ we have that $X, v \in T_{x} M$. Then the vertical lift of $X$ to $N$ at $v$ is a vector which is tangent at zero to a curve.

$$
t \longrightarrow v+t X
$$


Observation 1.3. The following map

$$
T_{\pi(v)} M \ni X \longrightarrow X^{V} \in T_{v} N^{V}
$$

is an isomorphism for all $v \in N$ (cf. 2.2. for the definition of $T N^{V}$ ).

Let $X \in \chi(M)$. Then there is defined the complete lif $X^{c}$ of $X$ to $N$. The field $X^{\mathrm{c}}$ is uniquely determined by the following property: for all $f \in C^{\infty}(M)$ we have that

$$
X^{c}(\mathrm{i} d f)=\mathrm{i} d(X(f)) .
$$

Observation 1.4. If $\varphi_{t}$ is a local flow of $X$ on $M$ then

$$
v \longrightarrow d \varphi_{t}(v)
$$

is a local flow of $X^{c}$ on $N$ (cf. [CDL]).

2.2. Let $(M, g)$ be a pscudo-Riemannian structure on the manifold $M$. Then the tensor $g$ determines the Levi-Civita connection $\nabla$ on $M$.

The connection $\nabla$ induces a 1-form

$$
\omega^{\nabla} \in T^{*} N \otimes_{N} \pi^{-1} T M
$$

defined uniquely by the following equations

$$
\begin{aligned}
\omega^{\nabla}(d Y(X)) & =\nabla_{X} Y \\
\omega^{\nabla}\left(X^{V}\right) & =X
\end{aligned}
$$

for all vector fields $X, Y \in \chi(M)$. By $\pi^{-1} T M$ we denote the pull-back bundle of $T M$ along the projection $\pi: N \rightarrow M$. In the above formula $d Y(X)$ denotes the differential of $Y$ evaluated at $X$. More preciscly, if $\left(U,\left(x^{1}, \ldots, x^{m}\right)\right)$ is a chart on $M$ and $\left(\left.N\right|_{U^{\prime}},\left(x^{1}, \ldots, x^{m}, y^{1}, \ldots, y^{m}\right)\right)$ is the induced chart on $N$ then

$$
d Y(X)=\sum_{i=1}^{m} X^{i} \frac{\partial}{\partial x^{i}}+\sum_{i=1}^{m} \sum_{\alpha=1}^{m} X^{i} \frac{\partial Y^{\alpha}}{\partial x^{i}} \frac{\partial}{\partial y^{\alpha}}
$$

where $X=\sum_{i=1}^{m} X^{i} \frac{\partial}{\partial x^{i}}$ and $Y=\sum_{j=1}^{m} Y^{j} \frac{\partial}{\partial y^{j}}$. Then we define the vertical and horizontal subspaces of the bundle $T N \rightarrow N$ in the following way:

$$
\begin{aligned}
T N^{V} & =\left\{X^{V} \in T N \mid X \in T M\right\}=\operatorname{ker} d \pi \\
(T N)^{H} & \left.=\{Z \in T N] \omega^{\nabla}(Z)=0\right\} .
\end{aligned}
$$


It is well-know that $(T N)^{V}$ and $(T N)^{H}$ are smooth subbundles of $T N \rightarrow$ $N$ and that we have the following direct sum of vector bundles:

$$
T N=(T N)^{V} \oplus(T N)^{H} \text {. }
$$

This decomposition implies that

$$
d \pi:(T N)^{H} \longrightarrow T M
$$

is an isomorphism on the fibres.

Suppose that $X, v \in T M$ then the horizontal lift of $X$ to $T_{v} N$ is a vector $X^{H} \in T_{v} N$ such that

$$
\begin{aligned}
& \text { (1). } X^{H} \text { is horizontal; } \\
& \text { (2). } d \pi\left(X^{H}\right)=X
\end{aligned}
$$

It is clear that the conditions above define uniquely the horizontal lift of a vector. In a natural way the horizontal lift is extended to vector fields.

Observation 1.5. From the construction of the horizontal subbundle it follows that

$$
\begin{aligned}
(T N)_{v}^{H}= & \left\{d Y(X) \in T N \mid \forall X \in T_{\pi(v)} M\right. \\
& \text { and } \left.\forall Y \in \chi(M) \text { such that } \nabla Y_{x}=0\right\} \\
= & i m d Y_{x} \text { where } Y \in \chi(M) \text { and } \nabla Y_{x}=0 .
\end{aligned}
$$

The direct sum decomposition of $T_{v} N$ and the above identifications allow us to define maps $p^{H}$ and $p^{V}$ such that

$$
\begin{aligned}
& p^{H}: T_{v} N \longrightarrow T_{\pi(v)} M \\
& p^{V}: T_{v} N \longrightarrow T_{\pi(v)} M
\end{aligned}
$$

where $p^{H}$ is just $d_{v} \pi$ and $p^{V}$ is a composition of a projection from $T_{v} N$ onto $T_{v} N^{V}$ with the identification of this space with $T_{\pi(v)} M$ (cf. Observation 1.3). The maps $p^{H}$ and $p^{V}$ serve for construction of three symmetric bilinear forms

$$
\begin{aligned}
I & :=g\left(p^{H}(*), p^{H}(*)\right) \\
I I & :=g\left(p^{H}(*), p^{V}(*)\right)+g\left(p^{V}(*), p^{H}(*)\right) \\
I I I & :=g\left(p^{V}(*), p^{V}(*)\right)
\end{aligned}
$$

We may repeat the construction of these forms point by point to obtain global forms on $N$. The forms $I I, I+I I, I+I I I, I I+I I$ appear to be Riemannian or pscudo-Riemannian metrics on $N$. These metrics are studied in [YI]; an interesting exposition of this subject may be found also in [Ia].

There are the following relations between the lifts defined above: 
Proposition 1.6. Let $X, Y \in \chi(M)$ and $p \in M$. We also denote $v=X_{p}$. Then the following identities hold

$$
\begin{aligned}
Y_{v}^{c} & =Y_{v}^{H}+\left(\nabla_{X} Y\right)_{v}^{V} \\
d X\left(Y_{p}\right) & =Y_{v}^{H}+(\nabla Y X)_{v}^{V} \\
d X\left(Y_{p}\right) & =Y_{v}^{c}+[Y, X]_{v}^{V}
\end{aligned}
$$

(cf. also $[\mathrm{YI}]$ ).

Proof: (ii) let $d X(Y)_{v}=A+B$ where $A \in T_{v} N^{H}$ and $B \in T_{v} N^{V}$. Then

$$
Y_{p}=d \pi \circ d X(Y)=d \pi(A+B)=d \pi(A) .
$$

Since $d \pi$ restricted to $T_{p} N^{H}$ is an isomorphism then we get that $A=Y_{v}^{H}$. On the other hand we have that

$$
\left(\nabla_{Y} X\right)_{p}=\omega_{v}^{\nabla}\left(d X\left(Y_{p}\right)\right)=\omega_{v}^{\nabla}(A+B)=\omega_{v}^{\nabla}(B) .
$$

Since $\omega_{v}^{\nabla}$ is an isomorphism when restricted to $T_{v} N^{V}$ then it follows that $\left(\nabla_{Y} X\right)_{v}^{V}=B$. Hence (ii) follows.

We shall demonstrate (iii) using a chart $\left(U,\left(x_{1}, \ldots, x_{m}\right)\right)$ such that $p \in U \subset M$. Then we may express $X$ and $Y$ as the linear combinations of the standard basis

$$
X=\sum_{i=1}^{m} X^{i} \frac{\partial}{\partial x^{i}}, \quad Y=\sum_{i=1}^{m} Y^{i} \frac{\partial}{\partial x^{i}}
$$

where $X^{i}, Y^{j}$ are $C^{\infty}$-functions on $U$. The local coordinates on $M$ determine in a natural way the local coordinates $\left(\left.N\right|_{U},\left(x_{1}, \ldots, x_{m}, y_{1}, \ldots, y_{1}\right)\right)$. It easy to check that in this new local coordinates the following equalities hold:

$$
\begin{aligned}
d X\left(Y_{p}\right) & =\left(Y_{p}^{1}, \ldots, Y_{p}^{m},\left.\sum_{\alpha=1}^{m} \frac{\partial X^{1}}{\partial x^{\alpha}}\right|_{p} \cdot Y_{p}^{\alpha}, \ldots,\left.\sum_{\alpha=1}^{m} \frac{\partial X^{m}}{\partial x^{\alpha}}\right|_{p} \cdot Y_{p}^{\alpha}\right) \\
Y_{v}^{c} & =\left(Y_{p}^{1}, \ldots, Y_{p}^{m},\left.\sum_{\alpha=1}^{m} X_{p}^{\alpha} \cdot \frac{\partial Y^{1}}{\partial x^{\alpha}}\right|_{p}, \ldots,\left.\sum_{\alpha=1}^{m} X_{p}^{\alpha} \cdot \frac{\partial Y^{m}}{\partial x^{\alpha}}\right|_{p}\right)
\end{aligned}
$$

(for the second equality cf. [YI, p. 15]). Then it follows that

$$
d X\left(Y_{p}\right)-Y_{v}^{c}=[Y, X]_{v}^{V}
$$

and then (iii) follows. 
Equality (i) is a consequence of (ii) and (iii). In fact, from (iii) we get that

$$
Y_{v}^{c}=d X\left(Y_{p}\right)-[Y, X]_{v}^{V} .
$$

Then from (ii) and from the fact that $\nabla$ is torsionless we get that

$$
\begin{aligned}
Y_{v}^{c} & =Y_{v}^{H}+\left(\nabla_{Y} X\right)_{v}^{V}-[Y, X]_{v}^{V} \\
& =Y_{v}^{H}+\left(\nabla_{Y} X+[X, Y]\right)_{v}^{V} \\
& =Y_{v}^{H}+\left(\nabla_{X} Y\right)_{v}^{V} .
\end{aligned}
$$

This ends the proof of (i) and of the proposition.

1.3. If $\phi:\left(M_{1}, g_{1}\right) \rightarrow\left(M_{2}, g_{2}\right)$ is a smooth map between two pseudoRiemannian manifolds then the tension field of $\phi$ is defined as

$$
r(\phi)=\operatorname{trace}_{9}, \nabla d \phi .
$$

Then $\phi$ is called harmonic if the tension field vanishes. The equivalent definition of harmonicity of $\phi$ is that $\phi$ is a stationary point of the energy functional

$$
E(\phi)=\frac{1}{2} \int_{M_{1}} \operatorname{trace}_{g_{1}}\left(\phi^{*} g_{2}\right) \nu_{g_{1}} .
$$

By $\nu_{g_{1}}$ we denote the canonical measure on $M_{1}$ induced by $g_{1}$. If $M_{1}$ is not compact then the energy may be defined on its compact subsets. Then $\phi$ appears harmonic iff such energies defined on compact subsets are stationary with respect to the compactly supported variations. The function

$$
e(\phi)=\frac{1}{2} \operatorname{trace}_{g_{1}}\left(\phi^{*} g_{2}\right)
$$

is called energy density of $\phi$ (cf. $[\mathrm{K}]$ ). For more details about harmonic maps and techniques used in that theory of. $\left[\mathbf{E L}_{1}\right],\left[\mathbf{E L}_{2}\right]$.

\section{Energy densities}

In this part of the paper we shall consider the properties of energy densities associated with different symmetric tensors.

Let $(M, g)$ be a pseudo-Riemannian manifold and let $X \in \chi(M)$. We fix $p \in M$ and suppose that, $X_{p}=v$. By $E_{1}, \ldots, E_{m}$ we denote a local orthonormal frame. Then the energy density associated with $X$ : 
$(M, g) \rightarrow(N, I)$ is the following:

$$
\begin{aligned}
2 e_{I}(X)_{p} & =\operatorname{trace}_{g} I(d X(*), d X(*))_{v} \\
& =\sum_{i=1}^{m} I\left(d X\left(E_{i}\right), d X\left(E_{i}\right)\right)_{v} \cdot g\left(E_{i}, E_{i}\right)_{p} \\
& =\sum_{i=1}^{m} I\left(E_{i}^{H}+\left(\nabla_{E_{i}} X\right)^{V}, E_{i}^{H}+\left(\nabla_{E_{i}} X\right)^{V}\right)_{v} \cdot g\left(E_{i}, E_{i}\right)_{p} \\
& =\sum_{i=1}^{m} g\left(E_{i}, E_{i}\right)_{p}^{2} \\
& =m
\end{aligned}
$$

Similarily we calculate that

$$
\begin{aligned}
2 e_{I}(X)_{p} & =\operatorname{trace}_{g} I I(d X(*), d X(*))_{v} \\
& =\sum_{i=1}^{m} I I\left(d X\left(E_{i}\right), d X\left(E_{i}\right)\right)_{v} \cdot g\left(E_{i}, E_{i}\right)_{p} \\
& =2 \sum_{i=1}^{m} g\left(E_{i}, \nabla_{E_{i}} X\right)_{p} \cdot g\left(E_{i}, E_{i}\right)_{p}
\end{aligned}
$$

and

$$
2 e_{I H}(X)_{p}=\sum_{i=1}^{m} g\left(\nabla_{E_{i}} X, \nabla_{E_{i}} X\right)_{p} \cdot g\left(E_{i}, E_{i}\right)_{p} .
$$

It follows that for a given real number $t \in R$

$$
\begin{aligned}
e_{I}(t X) & =\text { constant }=\frac{m}{2} \\
e_{I I}(t X) & =t e_{H}(X) \\
c_{H}(t X) & =t^{2} e_{H}(X)
\end{aligned}
$$

If $M$ is compact then there are defined the energies

$$
\begin{aligned}
E_{I}(X) & =\int_{M} e_{I}(X) \nu_{g} \\
E_{I I}(X) & =\int_{M} e_{I I}(X) \nu_{g} \\
E_{I H}(X) & =\int_{M}^{e_{I H}(X) \nu_{g} .}
\end{aligned}
$$


Since $I, I I I$ are degenerated metrics on $N$ the quantities defined above are not classical energies. However they have the following properties:

$$
\begin{aligned}
E_{I}(t X) & =\frac{m}{2} \operatorname{vol}(M) \\
E_{I I}(t X) & =t E_{I I}(X) \\
E_{I I}(t X) & =t^{2} E_{I I}(X) .
\end{aligned}
$$

\section{The metric $I+I I I$ (the Sasaki metric)}

Harmonic vector fields $X:(M, g) \rightarrow(T M, I+I I I)$ were investigated by Ishihara (cf. $[\mathrm{I}]$ ). The tension field obtained by Ishihara is the following:

$$
\tau(X)=\left(\operatorname{trace}_{g} R(\nabla, X, X) *\right)^{H}+\left(\left(\operatorname{trace}_{g} \nabla^{2} X\right)^{v}\right.
$$

cf. also [CS]. The vector field trace $\nabla^{2} X$ is called the rough Laplacian and is denoted by $\Delta X$. In the case when $M$ is compact it was proved that $X$ is harmonic iff it is parallel (cf. [I]). In the proof there is used Bochner's theorem ([Y, p. 39]). We give below very simple proof of the theorern of Ishihara for compact manifolds.

Theorem 3.1. Let $(M, g)$ be a compact Ricmannian manifold and $X \in \chi(M)$. Then $X$ is harmonic with respect to Sasaki metric on TM if and only if $X$ is parallel.

Proof: Suppose that $X$ is harmonic and let consider the following variation of $X$

$$
M \times \mathbf{R} \ni(x, t) \longrightarrow t X_{x} \in T M .
$$

Since $X$ is a critical point of the energy functional we have that:

$$
\begin{aligned}
0 & =\left.\frac{d}{d t} E_{I+H}(t X)\right|_{t=1} \\
& =\left.\frac{d}{d t} E_{H I}(t X)\right|_{t=1} \\
& =\left.\frac{d}{d t} t^{2} E_{H I}(X)\right|_{t=1} \\
& =E_{H I}(X) .
\end{aligned}
$$

This implies that

$$
0=e_{H I}(X)=\operatorname{trace}_{g} g\left(\nabla_{*} X, \nabla, X\right)
$$

hence $\nabla X=0$.

If $X$ is paraflel then it is clear that the tension field of $X$ vanishes hence the vector field is harmonic. 


\section{Metrics $I I, I+I I$}

Let $(M, g)$ be a pscudo-Riemannian manifold. The Levi-Civita connections on $N=T M$ defined by the metrics $I I$ and $I+I I$ are the same. In fact this is the complete lift of the Levi-Civita connection $\nabla$ to $N$ (cf. Proposition 6.6, p. 45, Proposition 3.1, p. 149 [YI]). The complete lift of $\nabla$ we shall denote by $\nabla^{c}$. This connection is characterized in the following way: if $X, Y \in \chi(M)$ then

$$
\begin{aligned}
\nabla_{X^{v}}^{c} Y^{V} & =0 \\
\nabla_{X^{v}}^{c} Y^{c} & =\left(\nabla_{X} Y\right)^{V} \\
\nabla_{X^{c}}^{c} Y^{V} & =\left(\nabla_{X} Y\right)^{V} \\
\nabla_{X^{c}}^{c} Y^{c} & =\left(\nabla_{X} Y\right)^{c} .
\end{aligned}
$$

Since the Levi-Civita connections of metrics $I I$ and $I+I I$ coincide so do harmonic maps defined by these metrics. We shall calculate the tension field of a vector field $X \in \chi(M)$. Let $\left(E_{1}, \ldots, E_{m}\right)$ be a local orthonormal frame around the point $p \in M$ and let $\varepsilon_{i}:=g\left(E_{i}, E_{i}\right)_{p}$ for $i=1, \ldots, m$. Then applying Proposition 1.6 we get

$$
\begin{aligned}
\tau(X)_{p}= & \operatorname{trace}_{g}\left(\nabla^{c} d X\right)_{p} \\
= & \left.\sum_{i=1}^{m} \varepsilon_{i} \nabla_{E_{i}}^{c} d X\left(E_{i}\right)\right|_{v}-\left.\varepsilon_{i} d X\left(\nabla_{E_{i}} E_{i}\right)\right|_{v} \\
= & \sum_{i=1}^{m} \varepsilon_{i}\left(\nabla_{E_{i}}^{c}+\left[E_{i}, X\right]^{v}\left(E_{i}^{c}+\left[E_{i}, X\right]^{V}\right)\right. \\
& \left.-\left(\nabla_{E_{i}} E_{i}\right)^{c}-\left[\nabla_{E_{i}} E_{i}, X\right]^{V}\right)_{v} \\
= & \sum_{i=1}^{m} \varepsilon_{i}\left(\nabla_{E_{i}}\left[E_{i}, X\right]+\nabla_{\left[E_{i}, X\right]} E_{i}-\left[\nabla_{E_{i}} E_{i}, X\right]\right)_{v}^{V} .
\end{aligned}
$$

We would like to remark that in the second equation above wo consider the covariant derivative $\nabla^{c}$ along $X: M \rightarrow N$. Hence we are interested only in the values of the vector fields on the image of $X$. This justifies the application of Proposition 1.6. Since the conncction $\nabla$ is torsionless we have that for all $i=1, \ldots, m$

$$
\begin{aligned}
& \nabla_{E_{i}}\left[E_{i}, X\right]=\nabla_{E_{i}} \nabla_{E_{i}} X-\nabla_{E_{i}} \nabla_{X} E_{i} \\
& {\left[\nabla_{E_{i}} E_{i}, X\right]=\nabla_{\left(\nabla_{E_{i}}, E_{i}\right)} X-\nabla_{X} \nabla_{E_{i}} E_{2} .}
\end{aligned}
$$


We apply these formulas to computc $\tau(X)_{p}$.

$$
\begin{aligned}
\tau(X)_{p}= & \left(\sum_{i=1}^{m} \varepsilon_{i} \nabla_{E_{i}} \nabla_{E_{i}} X-\varepsilon_{i} \nabla_{E_{i}} \nabla_{X} E_{i}\right)_{v}^{V}+\left(\sum_{i=1}^{m} \varepsilon_{i} \nabla_{\left[E_{i}, X\right]} E_{i}\right)_{v}^{V} \\
& -\left(\sum_{i=1}^{m} \varepsilon_{i} \nabla_{\left(\nabla_{E_{i}} E_{i}\right)} X-\varepsilon_{i} \nabla_{X} \nabla_{E_{i}} E_{i}\right)_{v}^{V} \\
= & \left(\sum_{i=1}^{m} \varepsilon_{i} \nabla_{E_{i}} \nabla_{E_{i}} X-\varepsilon_{i} \nabla_{\nabla_{E_{i}} E_{i}} X\right)_{v}^{v} \\
& +\left(\sum_{i=1}^{m} \varepsilon_{i} \nabla_{X} \nabla_{E_{i}} E_{i}-\varepsilon_{i} \nabla_{E_{i}} \nabla_{X} E_{i}+\varepsilon_{i} \nabla_{\left[E_{i}, X\right]} E_{i}\right)_{v}^{V} \\
= & \left(\operatorname{tracc}_{g} \nabla^{2} X+\sum_{i=1}^{m} \varepsilon_{i} R\left(X, E_{i}\right) E_{i}\right)_{v}^{V} \\
= & \left(\Delta X+\operatorname{trace}_{g} R(X, *) *\right)_{v}^{V} .
\end{aligned}
$$

In the above formula $R$ denotes the curvature tensor of $\nabla$. Then applying Observation 1.3 we get the following proposition.

Proposition 4.1. Let $(M, g)$ be a pseudo-Riemannian manifold and let $T M$ be aquipped with the metric $I I$ or $I+I I$ then a vector feld $X \in \chi(M)$ is harmonic with respect to these metrics if and ondy if

$$
\Delta X+\operatorname{trace}_{g} R(X, *) *=0 .
$$

Let us observe that for any $Y \in \chi(M)$ we have that

$$
\begin{aligned}
g\left(\text { trace }_{g} R(X, *) *, Y\right) & =\operatorname{trace}_{3} g\left(R(*, X) Y_{,} *\right) \\
& =\mathcal{R}(X, Y)
\end{aligned}
$$

where $\mathcal{R}$ denotcs the Ricci tensor of $(M, g)$. Hence we have that.

Corollary 4.2. If $(M, g)$ is a pseudo-Riemantian manifold and TM is equipped with one of the metrics $I I$ or $I+I I$ then a vector ficld $X$ is harmonic iff

$$
g(\Delta X, *)+\mathcal{R}(X, *)=0 .
$$

Corollary 4.3. If $(M, g)$ is a compact pseudo-Riemannian manifold and $X \in \chi(M)$ is harmonic with respect to the metrics $I I$ or $I+I I$ then $E_{I I}(X)=0$. 
Proof: Let us consider the variation $(x, t) \rightarrow t X$ then

$$
0=\left.\frac{d}{d t} E_{I I}(t X)\right|_{t=1}=\left.\frac{d}{d t} t E_{I I}(X)\right|_{t=1}=E_{I I}(X) .
$$

Corollary 4.4. If $(M, g)$ is a pseudo-Riemannian manifold and $X$ is a Killing vector field then $X$ is harmonic with respect to the metrics II or $I+I I$.

Proof: If $X$ is a Killing vector field then

$$
\operatorname{div} X=g(\Delta X, *)+\mathcal{R}(X, *)=0,
$$

converse is true if $M$ is compact (cf. $[\mathrm{P}]$ ). Hence our corollary follows.

Corollary 4.5. If $(M, g)$ is a Riemannian manifold with Ricci tensor negatively semi-defined (i.e. for each $V \in \xi(M) \mathcal{R}(V, V) \leq 0)$. Then a vector field $X$ is harmonic with respect to the metric $I I$ or $I+I I$ if and only if $X$ is parallel. Moreover, if $\mathcal{R}$ is negatively defined (i.e. $\mathcal{R}(V, V)=0$ iff $V=0$ ) then zero sectionss are the only harmonic vector fields.

Proof: We shall apply methods used in $[\mathbf{P}]$. Suppose that $X$ is harmonic. We have the following Bochner's formula valid for all vector fields:

$$
2 g(\Delta X, X)+2 \operatorname{trace}_{g}(\nabla X, \nabla X)+\Delta g(X, X)=0 .
$$

Since

$$
g(\Delta X, X)=-\mathcal{R}(V, V) \geq 0
$$

we get that

$$
2 \operatorname{trace}_{g}(\nabla X, \nabla X)+\Delta g(X, X) \leq 0
$$

then $\Delta g(X, X) \leq 0$. On a compact manifold this implies that $\Delta g(X, X)=0$ and then $\operatorname{trace}_{g}(\nabla X, \nabla X)=0$, so $\nabla X=0$. We have also obtained that $\mathcal{R}(X, X)=0$. Hence if $\mathcal{R}$ is negatively defined then $X=0$.

Notation. If $\varphi: M_{1} \rightarrow M_{2}$ is a diffeomorphism then by

$$
\bar{\varphi}: \chi\left(M_{1}\right) \longrightarrow \chi\left(M_{2}\right)
$$

we denotc the isomorphism of the modules of vector fields such that $\tilde{\varphi}(X):=d \varphi \circ X \circ \varphi^{-1}$. The operator $\tilde{\varphi}$ extends on the tensors of an arbitrary type (cf. [KN, p. 28]). 
Observation 4.6. Let $(M, g)$ be a pseudo-Riemannian manifold and let $Y$ be a Killing vector field and $X$ a harmonic vector field. Then $[Y, X]$ is harmonic.

Proof: Let $\varphi_{t}$ be a local flow of $Y$. Since $\varphi_{t}$ are local isometrics then we have that

$$
\tilde{\varphi}_{t} \Delta X=\Delta \tilde{\varphi}_{l} X \text { and } \tilde{\varphi}_{t} \operatorname{trace}_{g} R(X, *) *=\operatorname{trace} g\left(\tilde{\varphi}_{l} X, *\right) *
$$

These equations and harmonicity of $X$ imply that

$$
\begin{aligned}
0 & =-\left.\frac{d}{d t} \tilde{\varphi}_{t}\left(\Delta X+\operatorname{trace}_{g} R(X, *) *\right)\right|_{t=0} \\
& =-\left.\frac{d}{d t}\left(\Delta \tilde{\varphi}_{t} X+\operatorname{trace}_{g} R\left(\tilde{\varphi}_{t} X, *\right) *\right)\right|_{t=0} \\
& =\Delta[Y, X]+\operatorname{trace}_{g} R([Y, X], *) * .
\end{aligned}
$$

Hence from Proposition 4.1 it follows that $[Y, X]$ is harmonic. It is clear that $[X, Y]$ is also harmonic since the multiplication by -1 is an isometry of $N$.

Example 4.7. If $M$ is a Riemann surface of genus greater than one then its universal covering of $M$ is a hyperbolic plane with the constant curvature equal to -1 . There is a group of deck transformations acting isometrically on $M$. One can project the Riemannian structure from its universal covering to $M$. In this way it is possible to construct a Riemannian metric on $M$ with curvature -1 . Then Ricci curvature of $M$ is negatively defined and hence the only harmonic vector fields are zero sections. The same construction of a compact Riemannian manifold with constant negative curvature can be done for ench dirnension.

\section{References}

[CS] CadDeo R., Sanini A., Metriche armoniche indotte da campi vettoriali, Rendiconti Seminario Sci., Università Cagliari, 57, fasc. 2 (1987).

[CDL] Cordero C.A., Dodson C.T.J., "Differential geometry of frame bundles," Kluwer Academic publishers, Dordrecht, 1989.

[EL $\left.{ }_{1}\right]$ Eells J., Lemaire L., "Selected topics in harmonic maps," C.B.M.S. Regional Conference Series 57, Amer. Math. Soc., Providence, 1983.

[Ia] IANUS S., Sulle strutture canoniche dello spazio fibrato tangente di una varietà riemanniana, Rendiconti dì Matematica, Università di Roma, VI, 6, fasc. 1 (1973), 75-96. 
[I] Ishilitara T, Harmonic sections of tangent bundles, J. Math. Tokushima Univ. 13 (1979), 23-27.

[KN] Kobayasiil S., Nomizu K., "Foundations of differential geometry," Interscience Publishers, N. York, 1963.

[K] KonderAK, J.J., On natural first order Lagragians, Bull. London Math. Soc. 23 (1991), 169-174.

[P] POOR W., "Differential geometric structures," McGraw-Hill, N. York, 1981.

[Y] Yano K., "Integral formulas in Riemannian geometry," M. Dekker, N. York, 1970.

[YI] YANo K., ISHiliara S., "Tangent and cotangent bundles," Marcel Dekker, N. York, 1973.

Politecnico di Bari

Dipartimento di Matcmatica

Campus Universitario

Via Re David

70125 Bari

ITALYY

Primera versió rebuda el 12 de Desembre de 1990, dartera versió rebuda el 5 de Desembre de 1991 